\title{
Retrospective study of Rougan Tongdu Tuina combined with point-pressing massage therapy on neurodevelopment in children with delayed motor development at very early stage
}

\author{
Limei Geng, Yang Yang \\ Department of Neonatology, The First Affiliated Hospital of Guizhou University of Chinese Traditional Medicine, Guiyang, China \\ Contributions: (I) Conception and design: Both authors; (II) Administrative support: Y Yang; (III) Provision of study materials or patients: Y Yang; \\ (IV) Collection and assembly of data: L Geng; (V) Data analysis and interpretation: Both authors; (VI) Manuscript writing: Both authors; (VII) Final \\ approval of manuscript: Both authors. \\ Correspondence to: Yang Yang. Department of Neonatology, The First Affiliated Hospital of Guizhou University of Chinese Traditional Medicine, 71 \\ Bao Shan North Road, Guiyang 550001, Guizhou, China. Email: 523483997@qq.com.
}

Background: Particular factors before and after delivery can cause neurological damage to the fetus or newborn. Rougan Tongdu Tuina combined with point-pressing massage therapy (RTPM) can effectively stimulate the meridians and acupoints, and achieve the therapeutic effects of refreshing, resuscitating, and strengthening the brain and improving cognition. In this study, RTPM was used in the neonatal period, and quality evaluation results of general movements (GMs) were used to monitor the effect of this method on neurodevelopmental outcomes of children.

Methods: Children who were born in the First Affiliated Hospital of Guizhou University of Traditional Chinese Medicine from 1 March 2017 to 29 February 2020 were retrospectively enrolled. All newborns were assessed for the quality of GMs. Children in the RTPM group received RTPM, and the control group received conventional massage and point-pressing. A 20-day period constituted a round of treatment, each round was 20 days apart, and there were 3 rounds in total. The study participants received regular outpatient follow-ups, and clinical neurological examinations and Gesell development scales (GDS) assessment results were used to determine the outcome of motor development.

Results: A total of 96 newborns with abnormal GMs were retrospectively included, including 57 (59.4\%) males and 39 (40.6\%) females. There were 83 cases (86.5\%) of premature infants and 85 cases $(88.5 \%)$ of low birthweight infants, respectively. The Apgar score at $1 \mathrm{~min}$ after birth was rated 3-10 points, with an average of 7.4 points. The evaluation of GMs revealed that the indicators in the RTPM group were significantly better than those in the control group, and the number of children with abnormal GMs after each round of treatment was significantly reduced compared with the control group. At 6 months, the GDS score of the RTPM group was better than that in the control group. After the intervention, the total effective rate of the RTPM group was $92.2 \%$, which was significantly higher than the $75.6 \%$ of the control group $(\mathrm{P}<0.05)$.

Conclusions: The application of RTPM in the neonatal period can significantly improve the neurodevelopment of children.

Keywords: Tuina; point-pressing; motor development retardation; neurodevelopment

Submitted Sep 22, 2021. Accepted for publication Dec 17, 2021.

doi: $10.21037 / \mathrm{tp}-21-558$

View this article at: https://dx.doi.org/10.21037/tp-21-558 


\section{Introduction}

Rosenbaum et al. found that the perinatal period is an important phase in the process of embryonic development. Neurological damage to the fetus or newborn may be caused by exposure to high-risk factors before and after delivery. Among them, hypoxia, infection, asphyxia, premature delivery, low weight, and hyperbilirubinemia are important factors that cause cerebral palsy, abnormal behavior, intellectual disability, epilepsy, and other diseases (1). In clinical practice, infants who have risk factors for growth and development disorders (especially brain development) during pregnancy, childbirth, and the neonatal period are called high-risk infants $(2,3)$. With the gradual adjustment of China's childbirth policy, the age of pregnant women has increased, and various high-risk factors have emerged, resulting in a significant increase in the incidence of motor impairment in children. The results of previous studies have indicated that developmental abnormalities of the brain are difficult to diagnose promptly by imaging examinations such as B-ultrasound, computed tomography (CT), and nuclear magnetic resonance (NMR) (4). Moreover, the separation of imaging and clinical manifestations in some children has caused excessive medical treatment (5-7).

Based on a large number of research results over the past 10 years, researchers have gradually posited the critical period of brain development and the plasticity and compensation theory of the brain, providing a theoretical basis for the early rehabilitation of high-risk children $(8,9)$. Children's neuropsychiatric activity is a comprehensive manifestation of the functional interaction of the nervous system. The age of $0-3$ years is the most vigorous stage of brain development. Abundant external environmental stimuli have an important impact on the structure and functional development of the brain. Early intervention in high-risk children can promote the impaired brain function and further effectively compensate in the process of maturation and differentiation. The earlier the intervention, the better the curative effect, the greater the possibility that the brain will be reshaped and repaired, and the more conducive to minimizing the risk of disability (10). Therefore, how to provide early treatment to children with brain injury in order to reduce their disability rate and improve their quality of life is an important issue.

At present, there are no specific drugs and methods for curing perinatal brain injury, and modern rehabilitation physiotherapy techniques, acupuncture, massage, Chinese medicine, and so on, are often used (9). For children with perinatal brain injury of different types and periods, treatment methods are adopted to optimize their motor, language, and cognition recovery (9). Neural stem cell therapy is still immature. Increasingly, studies have shown that early adherence to rehabilitation of children with perinatal brain injury is the most effective means of recovery (11). Rougan Tongdu Tuina combined with point-pressing massage therapy (RTPM) was summarized by researchers based on the law of children's neurodevelopment and combined with traditional Chinese meridian therapy experience. It can effectively stimulate the meridians and acupoints, and achieve rejuvenation and resuscitation by relaxing the tendons and activating the collaterals with the pressing of acupoints. It also provides the therapeutic effect of strengthening the brain and improving intelligence. The therapy is safe, non-invasive, and readily accepted by children and their families (12). In our previous studies, we have shown that RTPM has a significant clinical effect on high-risk children with motor developmental delay (12). Therefore, the purpose of this study was to observe the clinical efficacy of RTPM on the neurodevelopmental outcomes of infants with very early motor development delay. We present the following article in accordance with the STROBE reporting checklist (available at https://dx.doi.org/10.21037/tp-21-558).

\section{Methods}

\section{Study population}

This study was conducted retrospectively. Newborns born in the Obstetrics Department of the First Affiliated Hospital of Guiyang College of Traditional Chinese Medicine from 1 March 2017 to 29 February 2020 were enrolled. All newborns were assessed for the quality of general movements (GMs), and those with "abnormal results of GMs assessment" were regarded as high-risk children for children with motor development delay. According to the treatment method, they were divided into the RTPM group and control group. The RTPM group was given Rougan Tongdu Tuina combined with point-pressing massage therapy, and the control group was given conventional Tuina and point-pressing. The inclusion criteria were as follows: (I) gestational age $<37$ weeks; (II) birth weight $<2,500 \mathrm{~g}$; (III) birth Apgar score 0-7 (assessed 1 min after birth); (IV) newborns with intracranial hemorrhage, central nervous system infection, and other diseases; (V) newborns with hyperbilirubinemia (in line with the 4th edition of the diagnostic criteria for hyperbilirubinemia in practical 
neonatology); (VI) twins, multiple births; (VII) newborns of mothers with diabetes, hypertension, hyperthyroidism, liver disease, and kidney disease during pregnancy; (VIII) cesarean section delivery with medical indications. The exclusion criteria were as follows: (I) those who had obvious visual impairment and could not complete the motor development assessment; (II) those who were lost to followup; (III) those who died during hospitalization in the neonatal intensive care unit; (IV) those who had congenital malformations. All parents or guardians of newborns signed an informed consent form. This study was approved by the Ethics Committee of the First Affiliated Hospital of Guizhou University of Chinese Traditional Medicine (No.: K2016-006). All procedures performed in this study involving human participants were in accordance with the Declaration of Helsinki (as revised in 2013).

\section{Newborn assessment}

\section{GMs quality assessment}

Records of GMs: the assessment was carried out on the day after birth using the internationally standardized video assessment method and recorded with a digital video camera. When recording, the participant was in a supine position on an incubator, crib, or mat. Children were dressed standard hospital gowns, and maintained in an active alert state. Attention was given to avoid crying, irritability, continuous hiccups, or the use of a pacifier. Recording lasted 5-10 min. Each infant was generally recorded 1-2 times, and the frequency of GMs assessment was appropriately adjusted according to the actual situation. The assessment was completed by 2 investigators who were certified in the rehabilitation training course of the Pediatric Hospital Affiliated to Fudan University. During the evaluation, the video audio signal was turned off, and the evaluator used Gestalt perception to evaluate the performance of each GMs, distinguishing between normal and abnormal subcategories. The GMs evaluation results were included as follows: the twisting movement phase: when there were multiple GMs records, the last evaluation result within 48 weeks of age was used as the result, and when there was only one GMs evaluation, that single time was used as the result. Principles for inclusion in the uneasy stage were as follows: if there were 1 or more uneasy movements in multiple GMs assessments, the result was classified as normal $(\mathrm{F}+)$, if the evaluation continuously lacked uneasy movements, the result was classified as abnormal (F-).

\section{Judgment of motor development outcome}

Regular outpatient follow-ups were performed on the participants, and GMs evaluation was performed after each round (20 days) of treatment. After the treatment, the children were evaluated by the Gesell development scales (GDS) at 3 and 6 months after birth respectively, as the assessment of the final motor development outcome. The outcomes were defined as follows: significantly effective, total exercise performance score increased by $\geq 15$ points; effective, total exercise performance score increased by 10 14 points; and invalid, exercise performance score increased by $<10$ points (13). The total effective rate was calculated as: (number of significantly effective cases + number of effective cases)/total cases in each group * $100 \%$. The neurological examination and the diagnosis of cerebral palsy were completed by the physician, and the Gesell assessment was conducted by the physician or assessor. "Abnormal results in GMs evaluation" were taken as diagnostically positive, "normal GMs evaluation results" as diagnostically negative, and "the outcome of motor development over 6 months of age determined by follow-up is normal" was taken as the main diagnostic criteria of follow-up outcome. Disease-free persons, as determined by the diagnostic gold standard, referred to those with a normal outcome of motor development.

\section{Treatment}

After inclusion, children were divided into the RTPM group and control group as described above. Regarding intervention time, the first GMs quality assessment was performed within 1 week after the birth of the newborn. If there was any abnormality, the intervention was started immediately. The intervention time involved 20 days as a round of treatment, with each round separated by 20 days, to a total of 3 rounds per participant.

\section{Children in the control group were given routine massage and acupuncture}

(I) Set acupoints were used for treatment of the upper limbs, lying in the supine position, as follows: Jianyu (LI15), Binao (LI14), Quchi (LI11), Chize (LU5), Shousanli (LI10), Waiguan (TH5), Lieque (LU7), and Hegu (LI4). The main techniques included pinch, push, knead, roll, squeeze, pressing, patting, tapping, and rubbing; (II) set acupoints were also used on the back of the lower limbs, in the prone position: Feishu (BL13), Ganshu (BL18), Weishu (BL21), Pishu (BL20), Mingmen (GV4), Yaoyangguan (GV3), 
Baliao (8 sacral foramina), Huantiao (GB30), Juliao (GB29), Chengfu (BL36), Weizhong (BL40), Chengshan (BL57), Feiyang (BL58), Shenmai (BL62), Zutaiyang, along the first lateral line of the bladder meridian, and the posterior part of the lower limbs. The administration method was as follows: (i) push and press the first lateral line of the Governor Vessel and Foot Taiyang Bladder Meridian and the posterior part of the lower extremity with one palm and heel; (ii) pinch and roll the posterior part of the lower extremity; (iii) using patting, tapping, or rolling on the lower back and the back of the lower limbs; the above 3 methods were repeated from top to bottom, repeated 3-5 times; (iv) using the palm rub method to act on the Du Channel and the first lateral line of the bladder meridian of foot taiyang, and the back of the lower limbs. Diathermy was considered appropriate; for pediatric chiropractic; (v) the above acupoints were pressed with the fingers for about half a minute per acupuncture point; (III) the following acupoints on the front, inner, and outer parts of the lower limbs were used, with the patient in the supine position: Tiguan, Heding, Knee, Yangling Spring (GB34), Zusanli (ST36), Sanyinjiao (SP6). For the front, inner, and outer parts of the lower limbs, the main techniques included pinch, push, knead, squeeze, press, pat, and rub. A slightly stronger stimulus was applied during massage to improve muscle tension.

\section{Children in RTPM group were given RTPM}

The following sequences were applied to RTPM group participants: (I) liver-softening massage and points pressing therapy: massage the meridian along the direction of flow of the liver meridian, and knead the Taichong (LR3) and Xingjian (LR2) points. (II) RTPM: Tapping: Tap the acupoints to relax the Dumai, from Changqiang (GV1) to Dazhui (GV14), 6 times once; Knead: from Changqiang to Dazhui point by point, press and rub each point 9 times and then push to the top acupuncture point, that is "kneading nine and pushing one"; Saw ridge: cross and push every 2 spinous processes horizontally, cross and push one by one from the Changqiang point to the end of the Dazhui point; Pinch the spine: press the radial edge of the thumb with both hands against the skin, Press the thumb and middle fingers forward, lift up the skin with 3 fingers at the same time, twist upwards from the Guiwei to the Dazhui point, twist 3 times and lift again, that is, "pinch three, lift one", 6 times once; Loosen the spine: relax the spine with the base of the palm, first rubbing and then scrubbing it gently 6 times; touch the fontanelle: lightly press the base of the right palm on the child's forehead, gather the roots of the thumb, middle, and ring fingers together, and place the base of the finger on the fontanelle, moving clockwise and counterclockwise with circular massage, slow, and gentle movements, to a total of 100 times in each direction.

\section{Statistical analysis}

Statistical analysis was performed using SPSS 22.0 statistical software (IBM Corp., Armonk, NY, USA). Quantitative data were tested for normal homogeneity, data conforming to normal distribution were expressed as mean \pm standard deviation, and $t$-test was used for comparison between groups. Qualitative data was expressed as a percentage, and comparisons between groups are performed by $\chi^{2}$ test or Fisher's exact test. A P value $<0.05$ indicated that the difference is statistically significant.

\section{Results}

\section{Baseline characteristics}

According to the inclusion and exclusion criteria, 96 newborns with abnormal GMs assessment were included (Table 1), including 57 males (59.4\%) and 39 females (40.6\%). Among these children, there were 83 (86.5\%) premature babies and $85(88.5 \%)$ low birthweight babies respectively. Apgar was scored as $3-10$ points, with an average of 7.4 points. Among those with abnormal GMs assessment, there were more cases with gestational ages of 31 and 32 weeks. There were 81 cases $(84.4 \%)$ of mothers experiencing pregnancy complications, $72 \%$ were second child, 58 cases of cesarean section (60.4\%), and 55 cases of cesarean section with medical indications (57.3\%).

\section{Outcome}

Patients with abnormal GMs should be treated within 1 week after birth. Children in RTPM group and control group received the GDS evaluation at 6 months after birth. After the intervention, the indicators of children in the intervention group were significantly better than those in the control group (Table 2), and the number of children with abnormal GMs after each treatment was significantly reduced compared with the control group (Table 3). After the intervention, the total effective rate of the intervention group was $92.2 \%$, which was significantly higher than the 
Table 1 Comparison of baseline characteristics between therapy group and controlled group

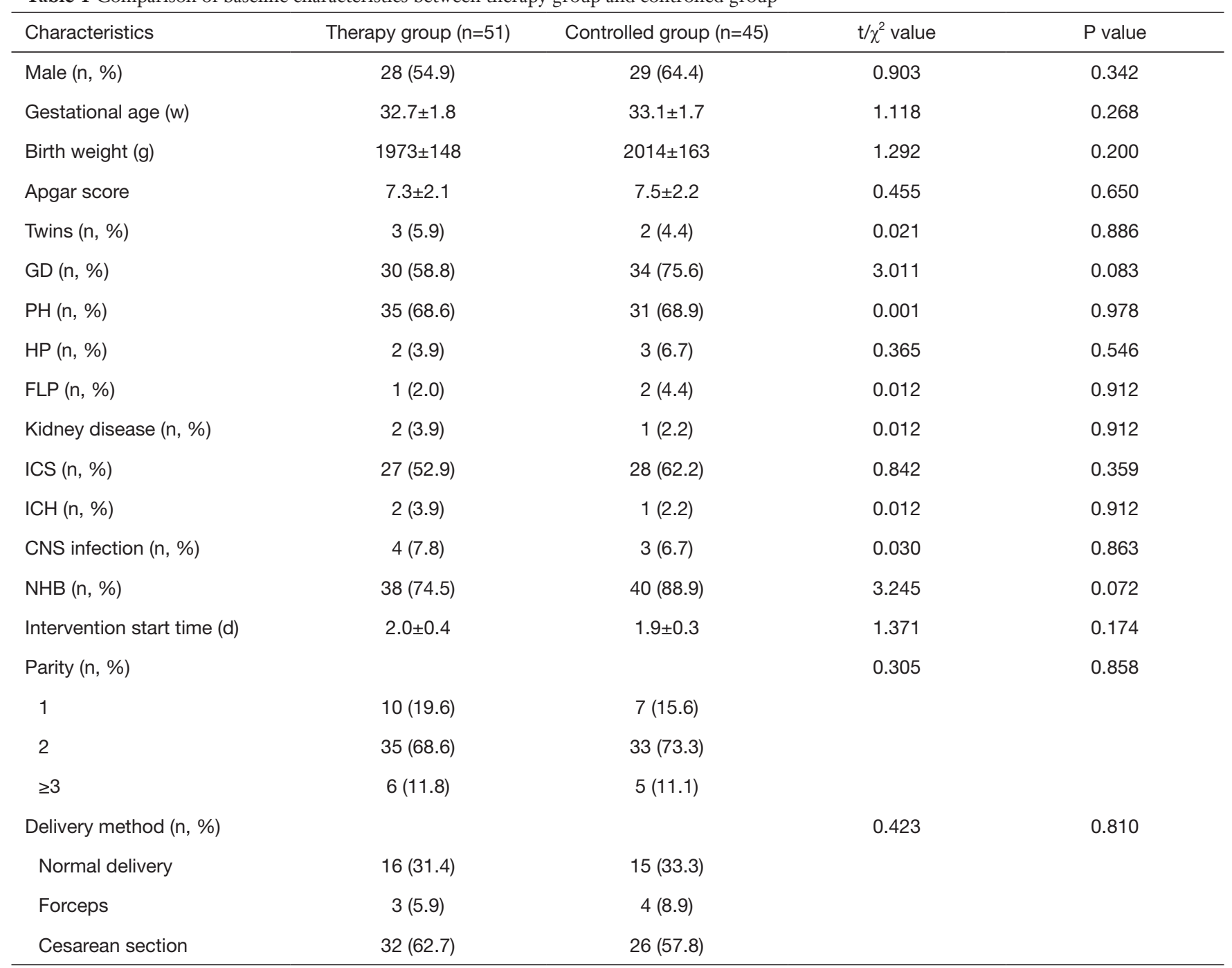

GD, gestational diabetes; PH, pregnancy hypertension; HP, hyperthyroidism in pregnancy; FLP, fatty liver of pregnancy; ICS, indicated cesarean section; $\mathrm{ICH}$, intracranial hemorrhage; $\mathrm{NHB}$, neonatal hyperbilirubinemia.

Table 2 Gesell development scales scores of the two groups before and six months after intervention $(\bar{x} \pm s)$

\begin{tabular}{lccc}
\hline Groups & Coarse movement & Fine movement & Total motor function \\
\hline Therapy group $(\mathrm{n}=51)$ & $88.0 \pm 7.2^{\mathrm{a} \star}$ & $88.3 \pm 5.6^{\mathrm{a} *}$ & $89.1 \pm 5.1^{\mathrm{a} \star}$ \\
Controlled group $(\mathrm{n}=45)$ & $71.33 \pm 5.2^{\mathrm{a}}$ & $70.5 \pm 6.1^{\mathrm{a}}$ & $72.3 \pm 5.6^{\mathrm{a}}$ \\
\hline
\end{tabular}

a , compared with scores before intervention, $\mathrm{P}<0.05$; ${ }^{*}$, compared with controlled group, $\mathrm{P}<0.05$.

75.6\% of the control group $(\mathrm{P}<0.05)($ Table 4$)$.

\section{Discussion}

Based on the previous research on children with early motor development delay (12), this study further showed that RTPM has a significant positive effect on the neurodevelopment of infants with very early motor development delay, with a total effective rate of $92.2 \%$, which can simultaneously improve gross motor function 
Table 3 Comparison of the number of cases with abnormal GMs assessment after 20 days of each treatment

\begin{tabular}{|c|c|c|c|c|}
\hline Characteristics & Therapy group $(n=51)$ & Controlled group $(n=45)$ & $\chi^{2}$ value & $P$ value \\
\hline 20 days after the second therapy (n, \%) & $12(23.5)$ & $35(77.8)$ & 28.154 & $<0.001$ \\
\hline 20 days after the third therapy (n, \%) & $3(5.9)$ & $17(37.8)$ & 14.746 & 0.0001 \\
\hline
\end{tabular}

GMs, general movements.

Table 4 Effectiveness in Therapy group [n (\%)]

\begin{tabular}{lccc}
\hline Characteristics & Therapy group $(\mathrm{n}=51)$ & Controlled group $(\mathrm{n}=45)$ & $\chi^{2}$ value \\
\hline Significantly effective $(\mathrm{n}, \%)$ & $38(74.5)$ & $15(33.3)$ & 16.509 \\
Effective $(\mathrm{n}, \%)$ & $9(17.6)$ & $19(42.2)$ & 0.0003 \\
Invalid $(\mathrm{n}, \%)$ & $4(7.8)$ & $11(24.4)$ & 4.998 \\
Total effective rate $(\mathrm{n}, \%)$ & $47(92.2)$ & $34(75.6)$ & 0.025 \\
\hline
\end{tabular}

and fine motor function. Our previous research showed that the earlier the intervention, the better the improvement of motor function (12). The children treated in this study were all neonates and were included in the study based on their assessment results on the day after birth. This study further shows that compared with ordinary massage, the effect of RTPM is superior.

Children's neurodevelopment has attracted increasing public attention, and there is an urgent need for technologies that can predict the outcome of neurodevelopment at an early stage, so that early intervention and treatment can reduce the occurrence of various sequelae. The quality assessment of GMs is currently one of the techniques that can predict the neonatal neurodevelopmental outcome at a very early stage (14-19). The technology for assessing GMs can predict abnormal brain function in infants, especially in terms of cerebral palsy. The future neurological developmental outcomes of infants can be effectively predicted through GMs assessment within 4 to 5 months after their birth, so as to effectively intervene as early as possible, and reduce the occurrence of sequelae of motor development delay $(2,20)$. We applied GMs assessment to the study of neonatal motor development, effectively distinguishing abnormal infants from normal infants (12). In this study, according to the results of the GMs evaluation, we intervened at the very early stage, that is, immediately after the birth of the child, and observed good therapeutic effects at the end of each treatment and at the evaluation 6 months after birth.

At present, more and more studies have shown that infants and young children with motor development delay should be identified as early as possible, and timely intervention should be applied. We know that exercise is closely related to the development of the advanced nervous system. The high-level nervous system controls motor functions, and exercise in turn stimulates the further development of the high-level nervous system, including intelligence, language, and other aspects (21-23), which has a profound impact on children's psychology and spirit $(24,25)$. Researchers have explored a variety of methods to assess the high risk of early motor development delay, such as GMs assessment and GDS (26), Griffiths development scale (27), and Alberta infant motor scale (AIMS) (28). Clinical practice has shown that these methods can effectively identify high-risk children. The purpose of early evaluation is to facilitate timely intervention. However, there are currently few effective treatments for motor delay, and such therapies are predominantly sports-based training and rehabilitation measures. Traditional Chinese medicine has certain effects in this situation, especially acupuncture, massage, and points pressing $(12,29-31)$. This method is based on the theory of traditional Chinese medicine. It is believed that it should clear the meridians and reduce muscular tension of the limbs; eliminate "liver stagnation", so that "liver blood" can be continuously supplied to the "heart", improve the "blood" supply of the "heart", and regulate mood; patting to relax the Dumai, stimulate the body's Yang meridian and qi blood, and improve brain and marrow function activities. The results of this study showed 
that this method can further improve the development of children's motor function compared with conventional massage. In addition to these methods, there are some other options. Mouse nerve growth factor and magnetic field stimulation have been showed to be useful on motor development, cerebral hemodynamics and biochemical metabolism in children with cerebral palsy $(32,33)$. Treatment like auditory stimulation also had positive effect on the motor development in children, but need further multi-center, randomized clinical trials to confirm (34).

This study has some limitations: it was a single-center study with a small sample size, and the treatment effect was closely related to the proficiency of doctors. In the future, we will conduct a multi-center large sample randomized controlled study in conjunction with multiple medical centers. Secondly, the follow-up time of this study was relatively short, and the long-term effect of this method cannot be evaluated at present. The next step will be to extend the follow-up time on the basis of a multi-center randomized controlled study to observe the children's motor function development at the age of 1 and 2 years.

\section{Acknowledgments}

Funding: This study was supported by the Guizhou Province Science and Technology Cooperation Project (Qiankehe LH [2016]7522).

\section{Footnote}

Reporting Checklist: The authors have completed the STROBE reporting checklist. Available at https://dx.doi. org/10.21037/tp-21-558

Data Sharing Statement: Available at https://dx.doi. org/10.21037/tp-21-558

Conflicts of Interest: Both authors have completed the ICMJE uniform disclosure form (available at https://dx.doi.org/tp21-558). Both authors report they received funding from Guizhou Province Science and Technology Cooperation Project (Qiankehe LH [2016]7522). The authors have no other conflicts of interest to declare.

Ethical Statement: The authors are accountable for all aspects of the work in ensuring that questions related to the accuracy or integrity of any part of the work are appropriately investigated and resolved. This study was approved by the Ethics Committee of the First Affiliated Hospital of Guizhou University of Traditional Chinese Medicine (No.: K2016-006). Written informed consent was taken from the patients' parents or legal guardians. All procedures performed in this study involving human participants were in accordance with the Declaration of Helsinki (as revised in 2013).

Open Access Statement: This is an Open Access article distributed in accordance with the Creative Commons Attribution-NonCommercial-NoDerivs 4.0 International License (CC BY-NC-ND 4.0), which permits the noncommercial replication and distribution of the article with the strict proviso that no changes or edits are made and the original work is properly cited (including links to both the formal publication through the relevant DOI and the license). See: https://creativecommons.org/ licenses/by-nc-nd/4.0/.

\section{References}

1. Rosenbaum PL, Walter SD, Hanna SE, et al. Prognosis for gross motor function in cerebral palsy: creation of motor development curves. JAMA 2002;288:1357-63.

2. Sadowska M, Sarecka-Hujar B, Kopyta I. Cerebral Palsy: Current Opinions on Definition, Epidemiology, Risk Factors, Classification and Treatment Options. Neuropsychiatr Dis Treat 2020;16:1505-18.

3. Guindos-Sanchez L, Lucena-Anton D, Moral-Munoz JA, et al. The Effectiveness of Hippotherapy to Recover Gross Motor Function in Children with Cerebral Palsy: A Systematic Review and Meta-Analysis. Children (Basel) 2020;7:106.

4. Clutterbuck G, Auld M, Johnston L. Active exercise interventions improve gross motor function of ambulant/ semi-ambulant children with cerebral palsy: a systematic review. Disabil Rehabil 2019;41:1131-51.

5. Moeschler JB, Shevell M; Committee on Genetics. Comprehensive evaluation of the child with intellectual disability or global developmental delays. Pediatrics 2014;134:e903-18.

6. Moeschler JB, Shevell M; American Academy of Pediatrics Committee on Genetics. Clinical genetic evaluation of the child with mental retardation or developmental delays. Pediatrics 2006;117:2304-16.

7. Kidokoro H, Anderson PJ, Doyle LW, et al. Brain injury and altered brain growth in preterm infants: predictors and prognosis. Pediatrics 2014;134:e444-53. 
8. Volpe JJ. Brain injury in premature infants: a complex amalgam of destructive and developmental disturbances. Lancet Neurol 2009;8:110-24.

9. Novak CM, Ozen M, Burd I. Perinatal Brain Injury: Mechanisms, Prevention, and Outcomes. Clin Perinatol 2018;45:357-75.

10. Volpe JJ. Dysmaturation of Premature Brain: Importance, Cellular Mechanisms, and Potential Interventions. Pediatr Neurol 2019;95:42-66.

11. Davidson JO, Dean JM, Fraser M, et al. Perinatal brain injury: mechanisms and therapeutic approaches. Front Biosci (Landmark Ed) 2018;23:2204-26.

12. Yang Y, Geng L, Cai J, et al. Clinical effect of Rougan Tongdu combined with point-pressing massage therapy on children at high risk of delayed motor development. Ann Palliat Med 2020;9:1174-9.

13. Huang JR, Zhang F, Wen FF, et al. Effect of Biofeedback Combined with Task-oriented Training on Hand Function, Gesell Scale Score and Balance Ability in Children with Spastic Cerebral Palsy. Sichuan Da Xue Xue Bao Yi Xue Ban 2020;51:428-33.

14. Pin TW, Yiu B, Wong T, et al. Development of Gross Motor Evaluation for Children Aged 18 to 42 Months. Dev Neurorehabil 2021;24:173-9.

15. Griffiths A, Toovey R, Morgan PE, et al. Psychometric properties of gross motor assessment tools for children: a systematic review. BMJ Open 2018;8:e021734.

16. Kakebeeke TH, Chaouch A, Knaier E, et al. A quick and qualitative assessment of gross motor development in preschool children. Eur J Pediatr 2019;178:565-73.

17. Veldman SLC, Santos R, Jones RA, et al. Associations between gross motor skills and cognitive development in toddlers. Early Hum Dev 2019;132:39-44.

18. Clutterbuck GL, Auld ML, Johnston LM. High-level motor skills assessment for ambulant children with cerebral palsy: a systematic review and decision tree. Dev Med Child Neurol 2020;62:693-9.

19. van Hinsbergh TMT, Elbers RG, van Furth MAM, et al. Longitudinal Association Between Human Parechovirus Central Nervous System Infection and Gross-Motor Neurodevelopment in Young Children. Pediatr Infect Dis J 2019;38:110-4.

20. Adde L, Helbostad JL, Jensenius AR, et al. Early prediction of cerebral palsy by computer-based video analysis of general movements: a feasibility study. Dev Med Child Neurol 2010;52:773-8.

21. Houwen S, Visser L, van der Putten A, et al. The interrelationships between motor, cognitive, and language development in children with and without intellectual and developmental disabilities. Res Dev Disabil 2016;5354:19-31.

22. Ross G, Demaria R, Yap V. The Relationship Between Motor Delays and Language Development in Very Low Birthweight Premature Children at 18 Months Corrected Age. J Speech Lang Hear Res 2018;61:114-9.

23. Iverson JM. Developing language in a developing body: the relationship between motor development and language development. J Child Lang 2010;37:229-61.

24. Peralta-Carcelen M, Schwartz J, Carcelen AC. Behavioral and Socioemotional Development in Preterm Children. Clin Perinatol 2018;45:529-46.

25. Choi B, Leech KA, Tager-Flusberg H, et al. Development of fine motor skills is associated with expressive language outcomes in infants at high and low risk for autism spectrum disorder. J Neurodev Disord 2018;10:14.

26. You J, Shamsi BH, Hao MC, et al. A study on the neurodevelopment outcomes of late preterm infants. BMC Neurol 2019;19:108.

27. Tso WWY, Wong VCN, Xia X, et al. The Griffiths Development Scales-Chinese (GDS-C): A cross-cultural comparison of developmental trajectories between Chinese and British children. Child Care Health Dev 2018;44:378-83.

28. Albuquerque PL, Guerra MQF, Lima MC, et al. Concurrent validity of the Alberta Infant Motor Scale to detect delayed gross motor development in preterm infants: A comparative study with the Bayley III. Dev Neurorehabil 2018;21:408-14.

29. Li LX, Zhang MM, Zhang Y, et al. Acupuncture for cerebral palsy: A meta-analysis of randomized controlled trials. Neural Regen Res 2018;13:1107-17.

30. Lu WP, Tsai WH, Lin LY, et al. The Beneficial Effects of Massage on Motor Development and Sensory Processing in Young Children with Developmental Delay: A Randomized Control Trial Study. Dev Neurorehabil 2019;22:487-95.

31. Hu CW, Wei YS, Du YM, et al. Effect of acupoint massage dominant early comprehensive intervention on the prognosis of premature infants with brain injury. Zhongguo Zhong Xi Yi Jie He Za Zhi 2014;34:1074-7.

32. He YN, Meng Y, Gao NN, et al. [Effect of injecting mouse nerve growth factor in different ways on motor development, cerebral hemodynamics and biochemical metabolism in children with cerebral palsy]. Zhongguo Zhen Jiu 2020;40:839-44.

33. Hollis A, Zewdie E, Nettel-Aguirre A, et al. Transcranial 
Static Magnetic Field Stimulation of the Motor Cortex in Children. Front Neurosci 2020;14:464.

34. Ben-Pazi H, Aran A, Pandyan A, et al. Auditory stimulation improves motor function and caretaker burden in children with cerebral palsy- A randomized double blind study. PLoS One 2018;13:e0208792.
Cite this article as: Geng L, Yang Y. Retrospective study of Rougan Tongdu Tuina combined with point-pressing massage therapy on neurodevelopment in children with delayed motor development at very early stage. Transl Pediatr 2021;10(12):3202-3210. doi: 10.21037/tp-21-558 